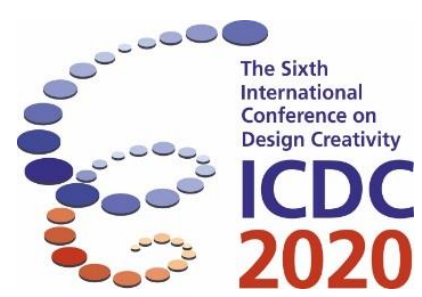

\title{
Opportunities with Uncertainties: The Outlook of Virtual Reality in the Early Stages of Design
}

\author{
Xinhui $\mathrm{Hu}$ and Georgi. V. Georgiev \\ Center for Ubiquitous Computing, University of Oulu, Finland
}

\begin{abstract}
Adopting immersive Virtual Reality (VR) technology in the early stages of design appeals to designers, as research has shown that immersing people in a virtual environment can efficiently elicit empathy and facilitate deeper understanding of out-group members. However, after exploring and synthesis literature across the fields of design, psychology, and neuroscience, the present study found the opportunities VR opens to be accompanied by uncertainties. In this study, we (1) identified the benefits of adopting immersive VR in the early design stage, such as enhancing empathy and promoting design equity, (2) discovered previously unrealised problems that VR may bring to the design process, especially potential biases owing to emotional connections, and (3) determined a future direction for relevant research: gaining deeper knowledge about operators' mental activities to mitigate biases and uncertainties.
\end{abstract}

Keywords: virtual reality, conceptual design, experience, empathy, human-centred design

\section{Introduction}

Gaining adequate and accurate knowledge of users' needs is a top concern for designers in the early stages of design, especially in the domain of human-centred design. In current practice, designers commonly exploit naturalistic methods, such as ethnographic observation and contextual inquiry, to build empathy for the intended users in order to better detect their true needs. However, due to the opaqueness and responsiveness of human mind, these methods will inevitably induce distortions and biases in the communication between designers and users, even they talk in the most professional and objective manner. Humans' information comprehension processes are mediated by complex and elusive mechanisms and are highly susceptible to individuals' experience and knowledge backgrounds. This is especially problematic when the users' experiences are too distant from designers' and even beyond their scope of understanding. On the other hand, the existence of designers in the naturalistic research process itself may constitute a strong confounding variable significantly changing the users' behaviour. Therefore, design researchers have been seeking an alternative method that will allow them to detour from existing issues.

Envisioned as an 'ultimate empathy machine', Virtual Reality (VR) technologies hold great promise to deepen designers' understanding of users. VR technologies are rapidly approaching their full potential of '[creating] a simulation of the physical world with which the operators could interact directly by means of the senses', as envisioned by their developer, Ivan Sutherland (Schroeder, 1993, p. 964). In the foreseeable future, fully developed VR technologies should enable designers to create a virtual environment that reliably mirrors the real-world scenarios experienced by users. Thus, new opportunities are emerging: to 'viscerally experience anything from another person's point of view' 
(Herrera et al., 2018, p. 2) and gain even deeper insights (Bollmer, 2017). More specifically, by immersing themselves in this virtual environment, designers will have the chance to experience, in an adequately similar context, what the users themselves experienced from the users' own perspectives. As shown in several studies, immersive perspective-taking experiences with VR enhance empathy and understanding of people with different identities (Gillath, McCall, Shaver, \& Blascovich, 2008; Herrera et. al., 2018; Schutte \& Stilinović, 2017). Moreover, unlike the physical world full of randomness, the virtual world is almost completely controlled and hence can eliminate confounding variables from the environment.

However, further investigation will be necessary before VR technology can be embraced in the early design stages (Hu et al., 2020). In-depth discussions regarding these unique opportunities, as well as possible concomitant uncertainties, are largely absent thus far. Technical limitations and incomplete understanding of the cognitive and emotional mental processes during VR operators' interaction with the virtual environment have both hindered explorations of this line of work. For example, while enhanced understanding and empathy for target users are considered a major strength of VR in supporting early design stages, the multi-faceted nature of empathy and perspective-taking process may entail confounding variables. Likewise, it is unclear whether the increased emotional association and self-other overlap phenomena observed in previous studies will significantly impact this process.

The answers to many crucial questions on exploiting VR in facilitating the early stages of design remain nebulous. Would strong emotional association influence designers' perceptions and observation? Would this connection impact the role of designers in the design process? Could VR be the ultimate empathy machine that designers have looked for? Probing these unknowns and exploring the answers motived this study. In working towards answering these questions, this study provides an outlook on how to apply VR technologies to facilitate the early stages of design by critically investigating the literature across disciplines, including design, psychology, information science, and neuroscience, and then identifying the opportunities and potential challenges in the design process. This study first presents a brief introduction to early design stages and VR-enhanced empathy studies and then discusses the potential benefits and problems of combining these research strategies.

\section{Early Stages of Design, User Research, and VR}

From observation to conceptual design, the early stages of design vary significantly in definition across design process models, yet most emphases ensuring adequate understandings of the needs and concerns of users. For instance, as shown in Figure 1, the famous design process models proposed by IBM, the Institute of Design at Stanford University, and the British Design Council all attach great importance to opportunity identification, observation, and problem definition (Design Council, 2015; IBM, 2018; Plattner, 2010). The underlying logic of these strategies is straightforward: without framing the right problems, designers will not approach the correct solutions.

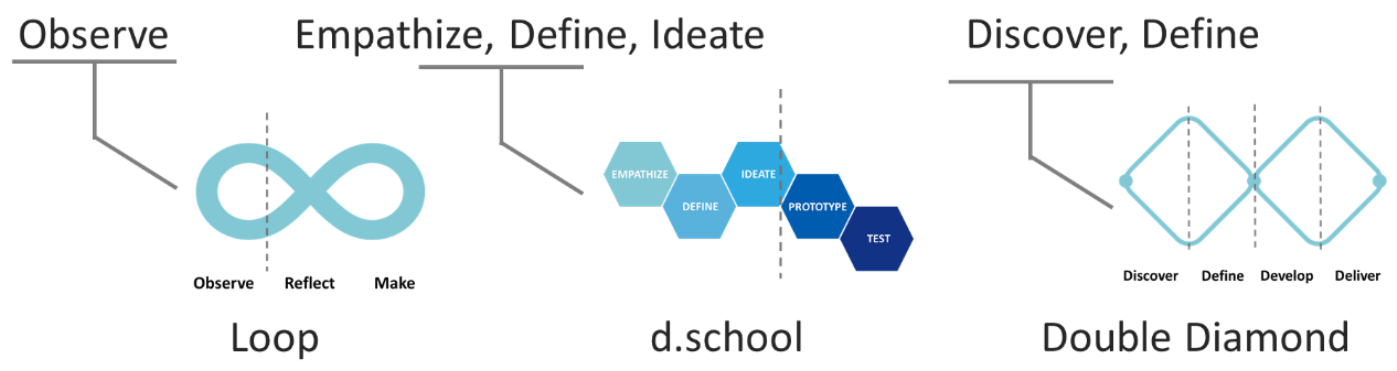

Figure 1. Early Stages of Famous Design Models

Devoting adequate effort to user research during the early stages of design has proven wise. Due to the opaqueness and responsiveness of human mental mechanisms, defining the right design problems to solve is much harder than it might at first appears. As users are constrained by their knowledge background and mindset, their narratives regarding their needs or problems tend to deviate significantly from the actual problems they encountered. To mitigate these potential biases, designers have exploited 
multiple naturalistic research methods, such as ethnographic observation and contextual inquiry, to complement users' self-reports about their needs. However, even the best-designed questions and the frankest user cannot guarantee accurate understandings. For example, information distortion during interactions with users and the limitation in empathetic scope are two inevitable problems with current user research strategies. Humans' memory retrieval and information comprehension processes are mediated by individuals' experience and knowledge frame, which leaves a vast space for mismatched understanding between designers and users. Moreover, in many cases the existence of designers itself serves as a confounding variable. As shown by the Hawthorne effect, merely realising others' attention is enough for people to significantly change their behavioural patterns. Thus, there is always the risk that the users may alter their behaviours when being treated as study subjects, thereby impairing the validity of the study. On the other hand, communication between designers and users may be hindered by their own 'empathetic horizon,' which describes 'the individual's range of understanding of user experiences in different contexts" (McDonagh-Philp \& Denton, 2008). If users' experiences are beyond the designers' scope of understanding, their interpretation of users' narratives can be erroneous and flawed. These error-prone factors are unwelcome by-products of the existing user research methods and hence are extremely hard to eliminate, without a new approach which allows designers to avoid these mental encumbrances. This is how promising VR technology is for catching the attention of designers. Leading human-tech interaction to a new dimension, VR is providing an alternate way of understanding users: seeing the world through the users' eyes. This saying used to be a pure metaphor, yet VR technology is turning this into reality. With the current head-mounted displays and interactive pointers, VR technology has facilitated the creation of highly immersive and interactive virtual environments in which users can achieve complex interactions. Moreover, when multi-sensory interaction can be achieved in the virtual environment, the specific features of users, such as height, strength, age, sex, and identities, can be embodied in the virtual avatar and experienced by the operator. In other words, designers can vividly sense the lives of users, including their subjective feelings, physical conditions, and even social interactions, in a fully developed VR environment, which will be especially beneficial for universal and inclusive design. Designers' empathetic horizons will be significantly expanded once these functions are available. To some extent, VR will be the ideal tool for the early stages of design, as it makes the emotional experience of others sensible to oneself by inhabiting another's body (Bollmer, 2017). It is also noteworthy that another unique benefit of VR is that the computer-generated virtual environment is almost completely controlled. Thus, designers can deliberately exclude confounding variables or distracting features while maintaining high ecological validity at the same time (Gillath et al., 2008). Thereby, VR can deepen designers' understanding of users and provide support in the design problem-definition and ideation stages.

The prospects of VR for facilitating user research in early stages of design has been partially validated by recent research. For example, the sensory experiences VR provides have been shown to promote the development of deeper understanding of others and empathy, especially for out-group members. Herrera and colleagues (2018) have found that immersive VR experience is more effective in eliciting empathy and establishing understanding of out-group members, especially compared to traditional imaginationbased perspective-taking tasks or non-immersive interactive experience (like first-person desktop video games). They also found that the empathetic effect of VR experiences persists longer and leads to more supportive behaviours. Furthermore, Yee and Bailenson (2006) demonstrated that immersive VR-based perspective-taking experience is a reliable method of reducing negative stereotypes associated with outgroup members. Because the understanding of others that users gain from the VR interaction is experience-driven, operators do not need to externalise, and at the same time reinforce, their stereotypes before attempting to eliminate them. These findings are encouraging, since the limited empathetic horizon and wrong knowledge of users are exactly the issues that bother designers.

\section{Probing the Uncertainties}

However, embracing VR in the design process without further discussion may be rash. Based on the existing knowledge, at least the following issues have been identified as potentially having strong influences on the validity of VR implementation in early stage of design. Thus, we must learn more about these factors to accurately assess the effectiveness of this strategy. 


\subsection{Uncertainties of Cognitive Mechanisms}

\subsubsection{Empathy}

Enhancement of empathetic experience is considered one of the major strengths of VR-based user research, but it remains unknown whether the complex nature of empathy will induce uncertainties. Brain imaging evidence suggests that human empathy involves at least five dimensions, as shown in Figure 2, under the mediation of distinct neural pathways.

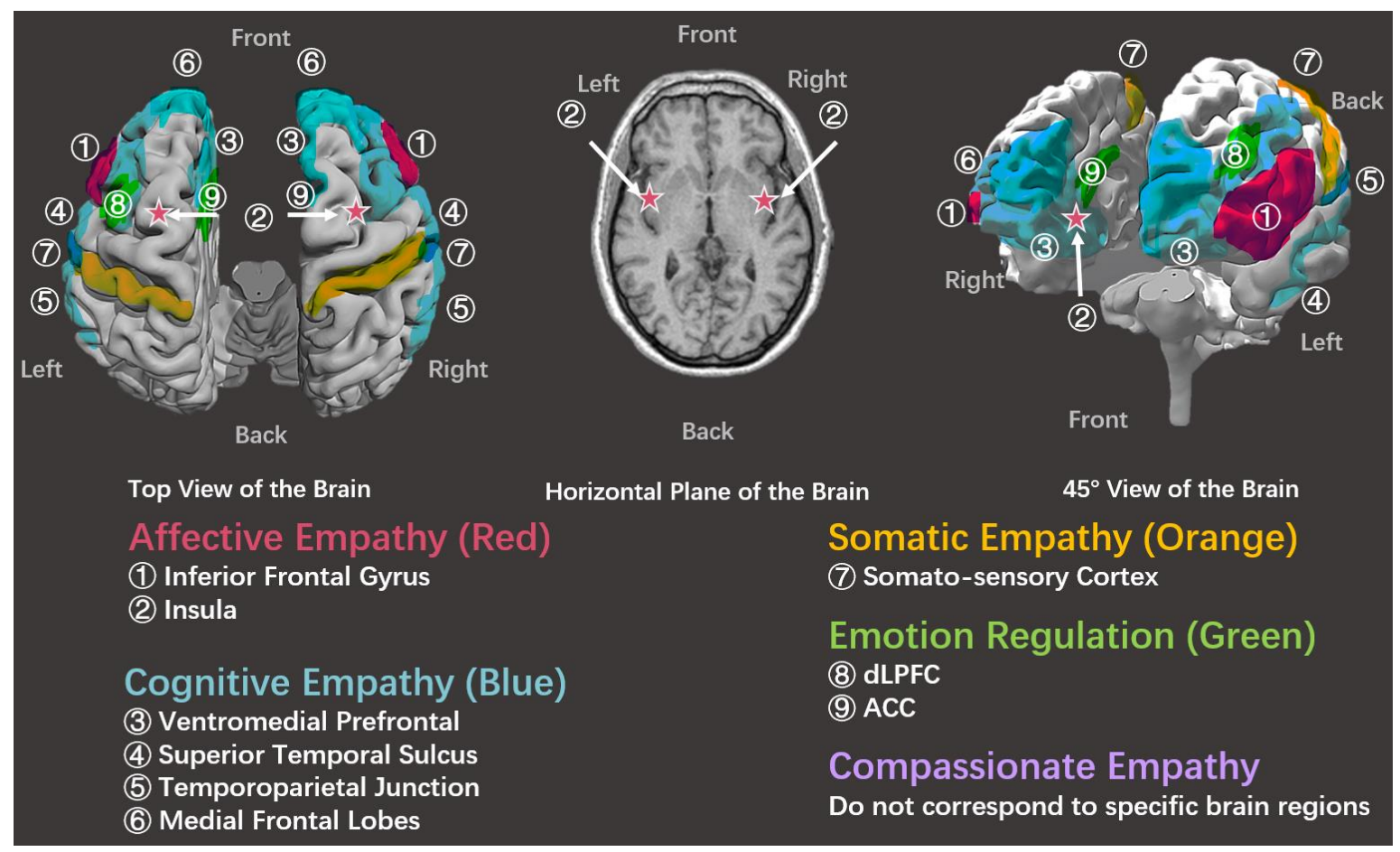

Figure 2. Neural Pathways of Different Types of Empathy

Moreover, each of these defined empathetic mechanisms evokes different corresponding cognitive functions. Affective empathy, 'I feel what you feel', allows people to resonate emotionally with others, which recruits more primitive and phylogenetically early emotion functions and hence tends to occur automatically or unconsciously (Bošnjaković \& Radionov, 2018; Shamay-Tsoory et al., 2009). Cognitive empathy, 'I understand what you feel', requires the ability to understand others' emotions by mentally taking their perspective and hence engages more advanced cognitive neural functions that require deliberate effort (Davis et al., 2004; Shamay-Tsoory et al., 2009). Besides these two major types, somatic empathy refers to the automatic neural-level mirroring processes to perceived emotional stimuli, especially to pain and sorrow (Bernhardt \& Singer, 2012; Price \& Dambha-Miller, 2019; Yagil, 2015). Compassionate empathy emphasis the benevolent behaviour-facilitating facet of empathy and has been regarded as an independent psychological state, which explains its inclusion in only a few models (Powell \& Roberts, 2017). Emotional regulation, which regulates humans' appropriate and flexible responses, relies on the executive neural circuits and enables the control of emotion (Decety, 2011; Eres, 2016). Which of these empathetic mechanisms will occur when adopting VR in early stages of design? How will they impact the understanding of users in this process? Will the virtual environment interfere with these mechanisms? These questions are important to VR implementation in design, as these underlying mechanisms will inevitably alter designers' understanding of users. But the answers are still absent.

\subsubsection{Perspective-taking}

Existing knowledge suggests that humans can adopt two perspective-taking strategies: imagine-self versus imagine-other (Batson et al., 1997). The former refers to people imagining how they themselves 
would perceive and feel if they were the observed person, while the latter refers to imagining how an observed person would perceive and feel in his or her situation. The difference between these strategies also influences the mental functions evoked. The imagine-other strategy tends to evoke mental representations of the observed person and altruistic motivation for prosocial behaviors, while the imagine-self strategy triggers self-related knowledge, personal stress, and egocentric motivation to help (Batson et al., 1997; Davis et al., 1996; Herrera et al., 2018). Whether the self-related memory retrieval and egocentric motivation will bias the design process remains unknown. Furthermore, a self-other merging phenomenon has been observed. As shown by Davis and colleagues, the observers will find 'their thoughts and feelings about an observed target to become, in some sense, more self-like.' (Davis et al., 1996, p.713) Due to technical limitations, the influence of perspective-taking on VR-based early stages of design is unclear, but its potential to blur the boundary between self and others and bias the designers is note-worthy.

\subsection{Uncertainties in Embodiment}

A successful embodiment process in immersive VR is typically signalled by the transfer of the operators' body schemas, the mental model of the body, from their physical bodies to their virtual avatars (Gorisse et al., 2017). However, when adopting VR in the early stages of design, merely coupling the physical body with the avatar is far from adequate. Associating the virtual avatar's identity with their body schema is crucial for designers to experience the lives of their target users and empathise with them. Current VR devices have fulfilled only the former task decently. In a study by Loon and colleagues (2018) of VR perspective-taking experiences and prosocial behaviours, they asked their participants to immerse themselves in the virtual environment and experience 'a day of the assigned avatar' from the avatar's perspective. The results suggested that the association of the identities of avatars with the participants' mental representations was unsuccessful. In other words, the participants did not actually experience the environment in another person's way as expected: they were still seeing others' worlds in their own eyes even if they viewed these worlds from the avatar's first-person viewpoint. Whether this situation is due to humans' intrinsic cognitive mechanisms or to limitations of VR technology remains unknown. The answer needs to be found before such technology can be implemented in design.

\subsection{Uncertainties in Social Interaction Rules}

Little has been learned as to whether the same social interaction rules in the real world will apply in the virtual environment due to the limitations in available technology. Multiple crucial questions that touch the core of the validity of this strategy have been proposed, to which researchers do not yet have good answers. For example, will the Hawthorne Effect also hold in the virtual world? Will the existence of people outside the virtual environment be intrusive from the VR operators' perspective? How will the operator balance the signals received from reality and the virtual environment? The answers are highly dependent on the performance of existing technology, so it may be too early to delve into this research theme, but these issues should be a matter of concern when discussing VR implementation strategies.

\section{Discussion: Outlook of VR in the Early Design Stages}

While the opportunities VR can bring to the early stage of design are unique, so are the uncertainties. How will these opportunities and uncertainties will trade off against each other when implementing VR technologies in the early design stage remains obscure. Thus, by synthesising the existing knowledge and the methods conceived by designers regarding this issue, we may arrive at an outlook on this issue that may help designers to evaluate the trade-offs before embracing this technology. The potential benefits and problems of exploiting VR in early design stages seem more obvious when they are listed together.

By synthesising the opportunities and uncertainties, at least three different strategies of utilising VR in early stages of design can be identified. 
Table 1. Opportunities versus Uncertainties

\begin{tabular}{|c|c|}
\hline Opportunity & Uncertainties \\
\hline $\begin{array}{c}\text { Enhance understanding of users (with first person } \\
\text { perspective experience) }\end{array}$ & $\begin{array}{c}\text { Limited understanding of empathy and the underlying } \\
\text { neural mechanisms }\end{array}$ \\
$\begin{array}{c}\text { Reduce stereotypes and wrong knowledge of out- } \\
\text { group members }\end{array}$ & $\begin{array}{c}\text { Ambivalent emotional reactions due to different } \\
\text { perspective-taking strategies }\end{array}$ \\
$\begin{array}{c}\text { Multi-sensory embodiment experience } \\
\text { Strike balance between experimental environment } \\
\text { and mundane realism }\end{array}$ & $\begin{array}{c}\text { Unclear effects of virtual-avatar embodiment } \\
\text { Incomplete knowledge of the social interaction rules } \\
\text { in the virtual environment }\end{array}$ \\
\hline
\end{tabular}

\subsection{Ergonomic Strategy}

The ergonomic strategy in utilising VR emphases detecting environmental, procedural, and physical issues from the users' perspectives. This strategy is based on three strengths of VR: (1) the possibility of replicating users' surroundings with high eco-validity, (2) multi-sensory embodiment, and (3) a precise record of the interaction data. Ergonomic research often requires a deep dive into the environment around users, which means that the presence of the researcher will be intrusive and the naturality of the observation cannot be guaranteed. On this issue, the ergonomic strategy provides a good alternative. In the simulated virtual environment, researchers can experience the interactive process of users, which is especially valuable when the research focuses on environmental and procedural issues. Meanwhile, in the computer-generated environment, the avatar's positions, motions, reaction time, and body gestures can all be precisely recorded and measured. Thus, with this technology, researchers can precisely replicate any experimental setting and specific procedure in the virtual environment (Gillath et al., 2008). Instant interaction with the virtual environment also makes prototyping and modelling much easier. The researchers can conveniently modify and test their design concepts and ideas in an environment that is adequately similar to the real-world scenario. Benefiting from the well-controlled immersive and interactive virtual environment, designers can gain a more accurate understanding of the users' ergonomic needs and integrate it into the conceptual design.

The only concern in adopting this method may be in determining the degree to which the virtual world should replicate the real world. It is not uncommon for seemingly irrelevant factors to be found play an important role. The elements to be included and excluded from the virtual environment need to be selected carefully.

\subsection{Empathetic Stream User Research}

The empathetic strategy emphasises the emotional and reflective aspects of user research. A central focus of this strategy is to 'approach the lives and experiences of users in order to increase the likelihood that the product or service designed meets the user's needs' (Kouprie \& Visser, 2009, p. 437), especially latent and unarticulated ones. When VR technology is able to (1) reliably replicate the actual world and (2) integrate different users' features into the virtual avatar, the social, emotional, interactive elements will also be simulated in the virtual environment and be experienced by the operators. In other words, this strategy is like a role-play process, during which the researcher can indeed experience what can happen when the users interact with such an environment, both physically and psychologically. These will provide effective support in mitigating the problems caused by the lack of direct experience, knowledge beyond the scope of understanding, and insufficient information.

However, while this strategy benefits more from VR's unique strengths, it is more susceptible to some uncertainties identified earlier. Whether the following uncertainties can be addressed will determine the validity of this strategy: (1) How well can VR operators immerse themselves in virtual avatars, especially when they have different identities? (2) How will the intrinsic complexity embedded in humans' empathy and perspective-taking influence this process? Will such cognitive functions occur together or in sequence? Would they intertwine and influence one another? (3) Furthermore, it is difficult to say whether the enhanced emotional connection with the user group is a positive thing for 
user research. The answers to these questions, which are crucial for offsetting potential biases and errors, remain unclear and hence require further studies.

\subsection{Design Equity Stream}

This strategy focuses on facilitating design equity and diversity with immersive VR. Compared to the empathetic strategy, the design equity strategy leans towards expanding the empathetic horizons to traditionally marginalised communities and user groups. In the book Design and Empathy (2003), the authors posed an interesting question: When designing for older adults, why does the focus tend to be on their special needs instead of their lifestyle and aspirations? This question reflects an array of intertwined social equity issues that are not limited to older adults and design...For example, inclusive design, which aims to embrace the special needs of everyone, has almost completely focused on meeting the ergonomic needs of the target design users. The 'aspirations and social needs that we all share regardless of age or capability' (Coleman et al., 2003, p. 480) are rarely taken into consideration. Why does the term 'special needs' seem to imply a problem to fix instead of a different lifestyle to be met? Why are people 'with special needs' often considered as 'with disabilities' instead of 'with different abilities'? When designing a kitchen for these marginalised groups, have designers focused too much on functional needs and too little on their aesthetic and social reflective values? Addressing these questions would lead us to a broad spectrum of social phenomena, whereas designers' stereotypes, insufficient knowledge of unfamiliar user groups, and limited empathic horizon definitely contribute to this problem.

The only problem may be the trade-offs between avoiding biases and reducing stereotypes, as both are results of the strong empathy and self-other merging caused by VR. Further research will be needed.

\section{Conclusion}

This study explored multiple facets of implementing immersive VR technology in the early design stage and made the following contributions: (1) synthesising the previously scattered knowledge on supporting the early stage of design with VR technologies, (2) revealing erstwhile unknown problems and concerns relevant to this research theme, (3) identifying the questions that must be answered to proceed with the research, and (4) pointing out the future direction of relevant research.

In conclusion, VR will bring unique benefits to designers in understanding design users' situations, detecting latent needs, enhancing experiment quality and ecological validity, and facilitating equitable and inclusive design, which also leaves more space for designers to exploit their creativity. However, the uncertainties underlying the VR implementation need to be investigated to prove the validity of this method. Moreover, adopting VR in the early design stage will significantly shorten the distance between designers and users and hence require designers to reconsider their roles in this process. Whether VR is the ultimate empathy machine that designers have sought requires further investigation. For the next step, we plan to carry out a series of empirical studies that will determine the psychological states and cognitive strategies of designers during VR-based user research as well as seek opportunities to offset the potential biases and uncertainties that accompany VR use.

\section{Acknowledgement}

This research has been partially financially supported by Academy of Finland 6Genesis Flagship (grant 318927). Thanks are due to Dr. Vijayakumar Nanjappan for the discussions on the revised paper.

\section{References}

Batson, C. D., Early, S., \& Salvarani, G. (1997). Perspective Taking: Imagining How Another Feels Versus Imaging How You Would Feel. Personality and Social Psychology Bulletin, 23(7), 751-758. https://doi.org/10.1177/0146167297237008

Bernhardt, B. C., \& Singer, T. (2012). The Neural Basis of Empathy. Annual Review of Neuroscience, 35(1), 123. https://doi.org/10.1146/annurev-neuro-062111-150536

Bollmer, G. (2017). Empathy machines. Media International Australia, 165(1), $63-76$. https://doi.org/10.1177/1329878X17726794 
Bošnjaković, J., \& Radionov, T. (2018). Empathy: Concepts, Theories and Neuroscientific Basis. Alcoholism and Psychiatry Research: Journal on Psychiatric Research and Addictions, 54(2), $123-150$. https://doi.org/10.20471/dec.2018.54.02.04

Davis, M. H., Conklin, L., Smith, A., \& Luce, C. (1996). Effect of perspective taking on the cognitive representation of persons: A merging of self and other. Journal of Personality and Social Psychology, 70(4), 713726. https://doi.org/10.1037/0022-3514.70.4.713

Davis, M. H., Soderlund, T., Cole, J., Gadol, E., Kute, M., Myers, M., \& Weihing, J. (2004). Cognitions Associated With Attempts to Empathize: How Do We Imagine the Perspective of Another? Personality and Social Psychology Bulletin, 30(12), 1625-1635. https://doi.org/10.1177/0146167204271183

Decety, J. (2011). Dissecting the Neural Mechanisms Mediating Empathy. Emotion Review, 3(1), 92-108. https://doi.org/10.1177/1754073910374662

Design Council. (2015, March 17). What is the framework for innovation? Design Council's evolved Double Diamond. Design Council. https://www.designcouncil.org.uk/news-opinion/what-framework-innovation-designcouncils-evolved-double-diamond

Eres, R. (2016). Scanning for empathy. Australasian Science, 37(2), 30.

Gillath, O., McCall, C., Shaver, P. R., \& Blascovich, J. (2008). What Can Virtual Reality Teach Us About Prosocial Tendencies in Real and Virtual Environments? Media Psychology, 11(2), 259-282. https://doi.org/10.1080/15213260801906489

Gorisse, G., Christmann, O., Amato, E. A., \& Richir, S. (2017). First- and Third-Person Perspectives in Immersive Virtual Environments: Presence and Performance Analysis of Embodied Users. Frontiers in Robotics and AI, 4. https://doi.org/10.3389/frobt.2017.00033

Herrera, F., Bailenson, J., Weisz, E., Ogle, E., \& Zaki, J. (2018). Building long-term empathy: A large-scale comparison of traditional and virtual reality perspective-taking. PLOS ONE, 13(10), e0204494. https://doi.org/10.1371/journal.pone.0204494

Hu, X., Georgiev, G. V., \& Casakin, H. (2020). Mitigating Design Fixation with Evolving Extended Reality Technology: An Emerging Opportunity. In Proceedings of the Design Society: DESIGN Conference, 1, 13051314. Cambridge University Press, https://doi.org/10.1017/dsd.2020.91

IBM. (2018, May 10). Learn the Enterprise Design Thinking Framework. https://www.ibm.com/design/thinking/page/framework/loop

Loon, A. van, Bailenson, J., Zaki, J., Bostick, J., \& Willer, R. (2018). Virtual reality perspective-taking increases cognitive empathy for specific others. PLOS ONE, 13(8), 0202442. https://doi.org/10.1371/journal.pone.0202442

McDonagh-Philp, D., \& Denton, H. (2008). User-centred design and the focus group: Developing the student designer's empathic horizons. Loughborough University. https://repository.lboro.ac.uk/articles/Usercentred_design_and_the_focus_group_developing_the_student_designer_s_empathic_horizons_/9345092

Nick Yee, \& Jeremy Bailenson. (2006, August 24). Walk a mile in digital shoes: The impact of embodied perspective-taking on the reduction of negative stereotyping in immersive virtual environments. Walk a Mile in Digital Shoes: The Impact of Embodied Perspective-Taking on the Reduction of Negative Stereotyping in Immersive Virtual Environments. Proceedings of PRESENCE 2006: The 9th Annual International Workshop on Presence, Cleveland, Ohio, USA.

Plattner, H. (2010). An introduction to design thinking process guide. In The Institute of Design at Stanford: Stanford.

Powell, P. A., \& Roberts, J. (2017). Situational determinants of cognitive, affective, and compassionate empathy in naturalistic digital interactions. Computers in Human Behavior, 68, 137-148. https://doi.org/10.1016/j.chb.2016.11.024

Price, A., \& Dambha-Miller, H. (2019). Empathy as a state beyond feeling: A patient and clinician perspective. Journal of the Royal Society of Medicine, 112(2), 57-60. https://doi.org/10.1177/0141076818790665

Schroeder, R. (1993). Virtual reality in the real world. Futures, 25(9), 963-973. https://doi.org/10.1016/00163287(93)90062-X

Schutte, N. S., \& Stilinović, E. J. (2017). Facilitating empathy through virtual reality. Motivation and Emotion, 41(6), 708-712. https://doi.org/10.1007/s11031-017-9641-7

Shamay-Tsoory, S. G., Aharon-Peretz, J., \& Perry, D. (2009). Two systems for empathy: A double dissociation between emotional and cognitive empathy in inferior frontal gyrus versus ventromedial prefrontal lesions. Brain, 132(3), 617-627. https://doi.org/10.1093/brain/awn279

Yagil, Y. (2015). The Physiology of Empathy: Theoretical and Practical Implications. In Encountering Empathy (p. 3). 